Ann. Geophysicae 16, 847-852 (1998) @ C EGS - Springer-Verlag 1998

\title{
Estimation of atomic oxygen concentrations from measured intensities of infrared nitric oxide radiation
}

\author{
A. S. Kirillov, G. A. Aladjev \\ Polar Geophysical Institute, Fersman str., 14, Apatity, Murmansk region, 184200, Russia
}

Received: 3 January 1996 / Revised: 31 July 1997 / Accepted: 16 September 1997

\begin{abstract}
The vibrational distribution of nitric oxide in the polar ionosphere computed according to the onedimensional non-steady model of chemical and vibrational kinetics of the upper atmosphere has been compared with experimental data from rocket measurement. Some input parameters of the model have been varied to obtain the least-averaged deviation of the calculated population from experimental one. It is shown that the least deviation of our calculations from experimental measurements depends sufficiently on both the surprisal parameter of the production reaction of metastable atomic nitrogen with molecular oxygen and the profile of atomic oxygen concentration. The best agreement with the MSIS- 83 profile was obtained for the value of surprisal parameter corresponding to recent laboratory estimations. The measured depression of level $v=2$ is obtained in the calculation that uses sufficiently increased concentrations of atomic oxygen. It is pointed out that similar measurements of infrared radiation intensities could be used to estimate the atomic oxygen concentrations during auroral disturbances of the upper atmosphere.
\end{abstract}

Keywords. Atmospheric composition and structure (airglow and aurora); Ionosphere (auroral ionosphere; ion chemistry and composition).

\section{Introduction}

Nitric oxide molecules have a valent electron and take an active part in the chemistry and vibrational kinetics of the atmosphere. In particular the cross sections of the inelastic collision of NO molecule have high magnitudes

Correspondence to: A. Kirillov in their interaction with other radicals. The rate coefficient of the interaction may be of the order of the gas-kinetic value (Smith, 1986). Since odd nitrogen is efficiently produced in the upper atmosphere during auroral precipitation, the concentrations of nitric oxide can be increased sufficiently influencing the chemical balance of the polar atmosphere. Moreover, as was shown in (Gordiets et al., 1982; Aladjev and Kirillov, 1992), nitric oxide plays a significant role in heat budget of the upper atmosphere. The infrared radiation in the 5.3- $\mu \mathrm{m}$ emission is important for the cooling process at the altitudes of $\mathrm{E}$ and $\mathrm{F}$ regions of the ionosphere. The purpose of the present work is to compare computed distributions of the vibrational population of nitric oxide in aurora with rocket experimental data of Rawlins et al. (1981) and to show the dependence of the calculations on the peculiarities of considered NO production and loss mechanisms and the composition of the upper atmosphere. One of the goals is to consider the possibility of estimating the order of the atomic oxygen concentration from experimental intensities of infrared radiation.

\section{Production and loss mechanisms}

Calculation of intensities of infrared nitric oxide radiation in the aurora requires knowledge of: (1) main production mechanisms of vibrationally excited nitric oxide in the atmosphere and quantum efficiencies of vibrational excitation to different levels in the excitations, (2) loss processes including spontaneous radiative transitions and vibrational relaxation in collisions with other atmospheric components. The primary sources of vibrationally excited NO in the auroral atmosphere have been discussed by Caledonia and Kennealy (1982), Aladjev and Kirillov (1993) and Kirillov and Aladjev (1995). These are: the chemical reactions of unexcited and metastable atomic nitrogen with molecular oxygen

$\mathrm{N}\left({ }^{4} \mathrm{~S}\right)+\mathrm{O}_{2} \rightarrow \mathrm{NO}(v)+\mathrm{O}$ 
$\mathrm{N}\left({ }^{2} \mathrm{D}\right)+\mathrm{O}_{2} \rightarrow \mathrm{NO}(v)+\mathrm{O}$

$\mathrm{N}\left({ }^{2} \mathrm{P}\right)+\mathrm{O}_{2} \rightarrow \mathrm{NO}(v)+\mathrm{O}$

where atoms $\mathrm{N}\left({ }^{4} \mathrm{~S},{ }^{2} \mathrm{D},{ }^{2} \mathrm{P}\right)$ are mainly produced in $\mathrm{N}_{2}$ dissociation and dissociative ionization by auroral electron impact or in ionic cycle of the auroral ionosphere, and TV-energy transfer in thermal collisions

$\mathrm{NO}(v=0)+\mathrm{O} \rightarrow \mathrm{NO}(v>0)+\mathrm{O}$.

The role of the reaction of fast nitrogen atom with $\mathrm{O}_{2}$ discussed by Sharma et al. $(1993,1996)$ as the mechanism of highly rotationally excited NO in the terrestrial thermosphere was investigated in (Lie-Svendsen et al., 1991) for auroral condition. It was shown that the mechanism is most likely unimportant for total NO abundance. Thus we presume here that the reaction of fast $\mathrm{N}\left({ }^{4} \mathrm{~S}\right)$ with $\mathrm{O}_{2}$ is of little consequence for the odd nitrogen chemistry in the lower thermosphere.

The product branching ratios

$P(v)=\frac{[\mathrm{NO}(v)]}{[\mathrm{NO}]}$

for $v=0-7$ of the reaction (1) were measured in Winkler et al. (1986). The branching ratios of the reactions (2a,2b) were measured in Kennealy et al. (1978), Green et al. (1984) and Rawlins et al. (1989). The experimentally observed $\mathrm{NO}(v)$ distributions were examined with the aid of surprisal theory (Bernstein and Levine, 1975; Nesbet, 1981) based on information theory. One of the successes of surprisal theory is the discovery that in many particular cases experimental or calculated finalstate populations $P(v)$ resulting from atom-molecular collisions take approximately the form

$P(v)=P^{0}(v) \exp \left(-\lambda f_{v}-\lambda_{0}\right)$

where $P^{0}(v)$ is a "prior" distribution for a vibrating rotator postulated to have zero information content or maximum entropy, $f_{v}$ is the fraction of the reaction's exoergicity appearing as vibrational energy in the product. Surprisal parameter $\lambda$ is a measure of the deviation of $P(v)$ from the statistically expected value.

The surprisal analysis of infrared emission spectrum of the NO $\Delta v=1$ bands in Kennealy et al. (1978) gave the surprisal parameter $\lambda_{1}=-2.1$ for the $\mathrm{O}\left({ }^{1} \mathrm{D}\right)$ branch of the reaction (2a). Similar estimations were obtained in Green et al. (1984). Rawlins et al. (1989) have used the technique which has been employed in Kennealy et al. (1978). The principal difference between their investigation and an earlier study is the more detailed analysis of the contribution of $\mathrm{N}\left({ }^{2} \mathrm{P}\right)+\mathrm{O}_{2}$ reaction in the observed $\mathrm{NO}(v)$ distribution. It is interesting to note that they obtained the parameter $\lambda_{1}$ after sufficient correction of "cold" distribution for lower vibrational levels (Fig. 8 in Rawlins et al., 1989). It was assumed as an upper bound that essentially all the $v=1$ population in the "cold" part is due to thermalized high rotationally excited NO. After scaling of the "hot" population and subtraction from "cold" part, Rawlins and co-workers have removed significant deviation on levels $v=1,2$ in "cold" population and obtained $\lambda_{1}=6.25$ for $\mathrm{O}\left({ }^{1} \mathrm{D}\right)$ branch (Figs. 9 and 10 in Rawlins et al., 1989). The final conclusions of Rawlins et al. (1989) are in significant disaccord with the results of Kennealy et al. (1978), and Green et al. (1984) showing much higher vibrational excitation of NO on levels $v=1-3$.

Main deactivation of high vibrational levels is produced by "radiation" because of the great magnitudes of the Einstein coefficients for these levels (Billingsley, 1976; Doroshenko et al., 1992) compared to the collisional loss processes. Since the Einstein coefficients for lower vibrational levels are smaller, the collisional loss processes can become important for "lower" vibrational levels. The dominant collisional loss process at the altitudes of the upper atmosphere appears to be the interaction with atomic oxygen:

$\mathrm{NO}\left(v_{i}\right)+\mathrm{O} \rightarrow \mathrm{NO}\left(v_{i}-\Delta v\right)+\mathrm{O}$

The relaxation of the molecule possessing orbital angular momentum differs sufficiently from LandauTeller adiabatic mechanism. Its interaction with collisional partners give rise to more than one electronic potential and the relaxation can occur in electronically non- adiabatic collision (Nikitin, 1974). A method like Landau-Zener approximation can be used to determine the possibilities of non-adiabatic transitions between the crossing surfaces.

When both the vibrationally excited molecule and its collision partner are free radicals there exists another mechanism for rapid relaxation. The two species may combine to form a strongly bound collision complex. The potential energy surface of the interaction has a deep "well" resulting in the production of an intermediate complex during the interaction. The lifetime of the complex exceeds periods of the complex vibrations sufficient for a randomization of the energy of internal excitation. The randomization means the transformation of vibrational energy into other kinds of energies during the existence of the complex. As discussed by Smith (1986), obviously the case is more typical for the interaction of radicals.

A statistical adiabatic model was developed by Quack and Troe (1974) to calculate the rate coefficients in the process via the formation of combined complex. The rate constant for the complex formation $k_{c}$ is unlikely to depend strongly on initial vibrational level $v_{i}$ of the molecule (Smith, 1986). Moreover, the experimental estimation of $\mathrm{NO}(v=1,2)$ relaxation on atomic oxygen at high temperatures in Glänzer and Troe (1975) has shown about equal magnitudes of rate coefficients for $v=1$ and 2. Quack and Troe (1977) have suggested that the rate constants for the decomposition of a triatomic complex to final vibrational level $v_{f}$ are proportional to $\left(E-E_{f}\right)^{n}$, where $E=E_{i}+3 / 2 k T$ is a thermally averaged total energy, $k$ and $T$ are Boltzmann constants and the temperatures, $E_{i}, E_{f}$ are vibrational energies of initial and final levels $v_{i}$ and $v_{f}=v_{i}-\Delta v, 1 / 2 \leq n \leq 3 / 2$. 
The room temperature value of the rate coefficient $k_{5}=6.5 \cdot 10^{-11} \mathrm{~cm}^{3} \mathrm{~s}^{-1}$ for the relaxation of $\mathrm{NO}(v=1)$ on atomic oxygen was measured by Fernando and Smith (1979). Using the value and the relation of $E_{i}$ and $E_{f}$ for anharmonic oscillator:

$\frac{E_{i}-E_{f}}{k}=2700 \cdot\left[\left(v_{i}-v_{f}\right)-2 x_{e} \frac{\left(v_{i}-v_{f}\right)\left(v_{i}+v_{f}-1\right)}{2}\right]$

where $x_{e}$ is the anharmonic constant of nitric oxide, we obtain the following expression for the process (5) in the case of $n=1$

$$
\begin{aligned}
& k_{5}\left(v_{i}, v_{f}\right)=7.4 \cdot 10^{-11} \\
& \times \frac{\frac{3}{2} T+2700 \cdot\left[\left(v_{i}-v_{f}\right)-2 x_{e} \frac{\left(v_{i}-v_{f}\right)\left(v_{i}+v_{f}-1\right)}{2}\right]}{\frac{3}{2} T\left(v_{i}+1\right)+2700 \cdot\left[\frac{v_{i}\left(v_{i}+1\right)}{2}-2 x_{e} \frac{\left(v_{i}-1\right) v_{i}\left(v_{i}+1\right)}{3}\right]} .
\end{aligned}
$$

The collisions with other atmospheric components $\mathrm{N}_{2}$ and $\mathrm{O}_{2}$ are inefficient in $\mathrm{NO}(v)$ relaxation at auroral altitudes because of small rate constants (Murphy et al., 1975; Green et al., 1982).

Since it is difficult very often to measure, in the laboratory, the rates of the interaction of radicals for different internal excitation of molecular reagent, an analysis of satellite or rocket experimental data on infrared emissions in upper atmosphere helps us to understand the peculiarities of the chemistry and vibrational kinetics of the radicals.

\section{Calculations and discussion}

Aladjev and Kirillov (1993) and Kirillov and Aladjev (1995) used a model to calculate the intensities of infrared emissions 5.3 and $2.7 \mu \mathrm{m}$ in the aurora. The theoretical estimations of energy efficiencies of the emissions were in agreement with rocket and aircraft data (Huppi and Stair, 1979; Rawlins et al., 1981; Reidy et al., 1982). The efficiencies mean the fractions of auroral electron energy deposited are those radiated in the 5.3 and $2.7-\mu \mathrm{m}$ emissions. The anomalous depression found by Rawlins et al. (1981) in the vibrational population of level $v=2$ of $\mathrm{NO}$ was reproduced theoretically using many quantum transitions $\Delta v=v_{i}$ in Eq. (5), and greater atomic oxygen concentration than that given by MSIS- 83 model. However the shape of the calculated populations in levels $v=1-6$ for altitudes 102 and $108 \mathrm{~km}$ did not agree with the data scans.

The observations of Rawlins et al. (1981) have included 10 scans passing through the Earth limb at payload altitudes of $96-125 \mathrm{~km}$. The vibrational distribution of nitric oxide in the aurora according to eight scans is presented in Rawlins et al. (1981). Here we would like to compare our model calculation with the data set, varying some input parameters of the model.

Our one-dimensional non-steady model of polar upper atmosphere is analogous to the model of Aladjev and Kirillov (1993), Kirillov and Aladjev (1995). The calculation of NO vibrational populations was made for auroral electron precipitation with a mean electron energy of $8 \mathrm{keV}$ and intensities of the molecular nitrogen ion band 391.4-nm about $100 \mathrm{kR}$. The concentration profiles of the main atmospheric constituents $\mathrm{N}_{2}$ and $\mathrm{O}_{2}$ are taken from MSIS-83. The scheme of ionospheric chemical reactions between main and minor components is taken from Torr and Torr (1982). The ionization, dissociation, dissociative ionization of $\mathrm{N}_{2}, \mathrm{O}_{2}$ and $\mathrm{O}$ by auroral electron impact are calculated using the method of "energetic costs" developed by Gordiets and Konovalov (1991). The model allows us to compute the concentrations of odd nitrogen $\mathrm{NO}, \mathrm{N}\left({ }^{4} \mathrm{~S},{ }^{2} \mathrm{D},{ }^{2} \mathrm{P}\right)$, ions $\mathrm{N}_{2}^{+}, \mathrm{O}_{2}^{+}, \mathrm{O}^{+}\left({ }^{4} \mathrm{~S},{ }^{2} \mathrm{D},{ }^{2} \mathrm{P}\right), \mathrm{NO}^{+}$, $\mathrm{N}^{+}$, thermal ionospheric electrons at the altitude interval $90-500 \mathrm{~km}$ and includes the processes of eddy and molecular diffusion.

In the first place, the branching ratios used in Kirillov and Aladjev (1995) were taken according to the values of Rawlins et al. (1989). Since the estimations of surprisal parameter $\lambda_{1}$ for $\mathrm{O}\left({ }^{1} \mathrm{D}\right)$ branch of the reaction (2a) by Kennealy et al. (1978) and Rawlins et al. (1989) have given different values $(-2.1$ and -6.25 , respectively), the parameter is varied in our calculation from -7 to -2 . The parameter for $\mathrm{O}\left({ }^{3} \mathrm{P}\right)$ branch used according to the magnitude of Rawlins et al. (1989). The branching ratios $P(v)$ of the reaction (2a) for considered interval of $\lambda_{1}$ calculated according to Eq. (4) are presented in Fig. 1.

Secondly, we suppose the decomposition of triatomic complex $\mathrm{NO}_{2}$ not only according to Quack and Troe (1977), but also consider the case of the preference of complete vibrational deactivation in Eq. (5) $\Delta v=v_{i}$. The values $n=1$ and 12 are consequently taken for the two cases.

Finally, we investigate the influence of atomic oxygen concentration on our calculated population. We use atomic oxygen concentrations given by model MSIS- 83 then multiply this by factors $\alpha=0.6-2$ and displace the profile by $\Delta h \mathrm{~km}$ :

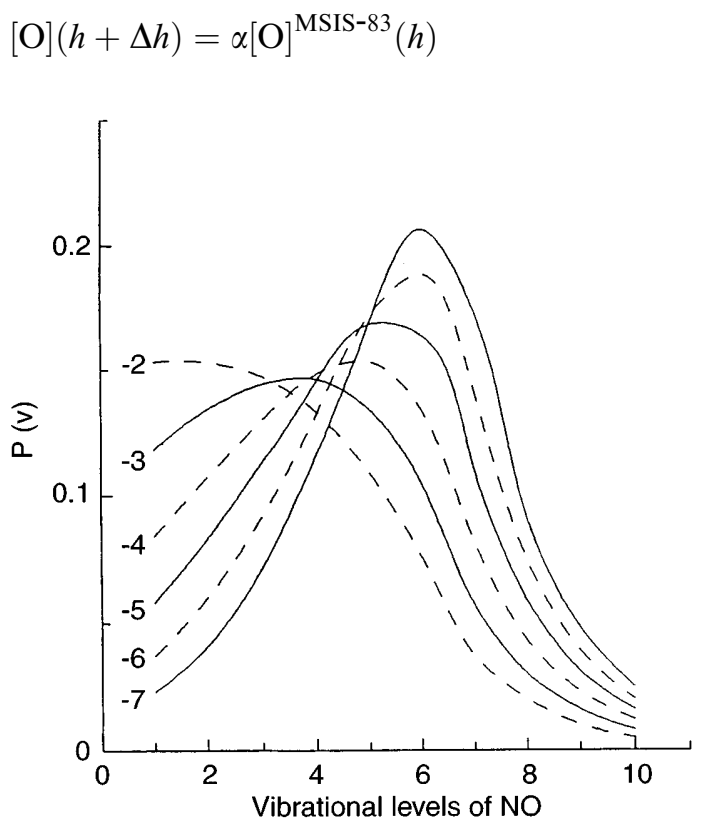

Fig. 1. The branching ratios, calculated according to surprisal theory, of the reaction (2a) for $\lambda_{1}=-7--2$ 
To compare our calculations with experimental results, we have chosen the distributions from the scans presented in Rawlins et al. (1981) minus the data for 74 scans and have normalized the sum of NO vibrational population $p_{\exp }(v)$ of the levels $v=2-6$ for every scan considered:

$\sum_{v=2}^{6} p_{\exp }(v)=1$.

Since the population of level $v=1$ is mainly conditioned by TV-excitation (Kirillov and Aladjev, 1995), it is not considered in the comparison. The computed distributions $p_{c a l}(v)$ were normalized in a similar manner.

The calculation has shown that the upward displacement of the profile of atomic oxygen concentration reduces the least-averaged deviation but the influence is negligible for $\Delta h \leq 4 \mathrm{~km}$. The following increase of $\Delta h$ leads to a sufficient rise of the averaged deviation in comparison with the case $0 \mathrm{~km} \leq \Delta h \leq 4 \mathrm{~km}$. The averaged deviation of the calculation from experimental data versus $\alpha$ for $\Delta h=4 \mathrm{~km}$ is shown in Fig. $2 \mathrm{a}, \mathrm{b}$ for $n=1$ and 12 , respectively. It is seen that the best
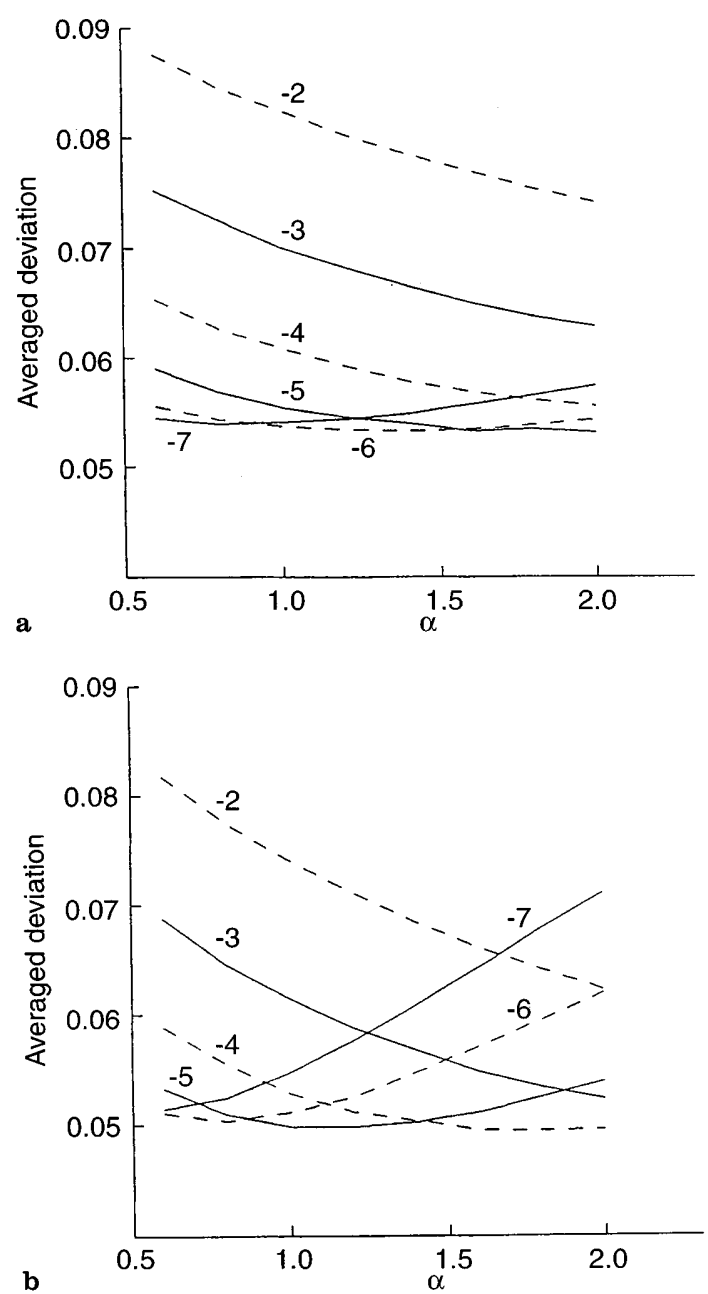

Fig. 2a,b. The averaged deviation of calculated NO populations from experimental data versus $\alpha$ for $\Delta h=4 \mathrm{~km}$ and $\lambda_{1}=-7--2$ : $\mathbf{a} n=1$, b $n=12$ agreement of the calculation with experimental data for $\alpha=1$ (insignificant displacement of MSIS-83 profile of [O]) takes place if the branching ratios $P(v)$ are calculated for $\lambda_{1}=-7--6$ for the case of $n=1$ and $\lambda_{1}=-6-5$ for the case of $n=12$.

To obtain the anomalous depression of level $v=2$ discussed by Rawlins et al. (1981) we have to presume in our calculation the atomic oxygen concentrations enhanced by a factor $2-3$ in comparison with MSIS- 83 profile. The enhanced concentrations are necessary to reduce the collisional lifetime of the level. The calculated populations of $\mathrm{NO}$ at the altitude $98 \mathrm{~km}$ for $n=1$ and $n=12$ are compared in Fig. 3a,b with the data of scan 137. The atomic oxygen concentrations of MSIS- 83 are enhanced by a factor of 2.5 . It is seen that anomalous depression of level $v=2$ and the decrease of the population with the rise of $v \geq 3$ can be obtained if we have taken the regime of the preference of complete vibrational deactivation in the reaction $(5)(n=12)$ and $\lambda_{1}=-4$ for the $\mathrm{O}\left({ }^{1} \mathrm{D}\right)$ branch of the reaction (2a). The error bars indicated in Fig. 3a,b are merely the statistical error in the average (Rawlins et al., 1981). The true uncertainty in the
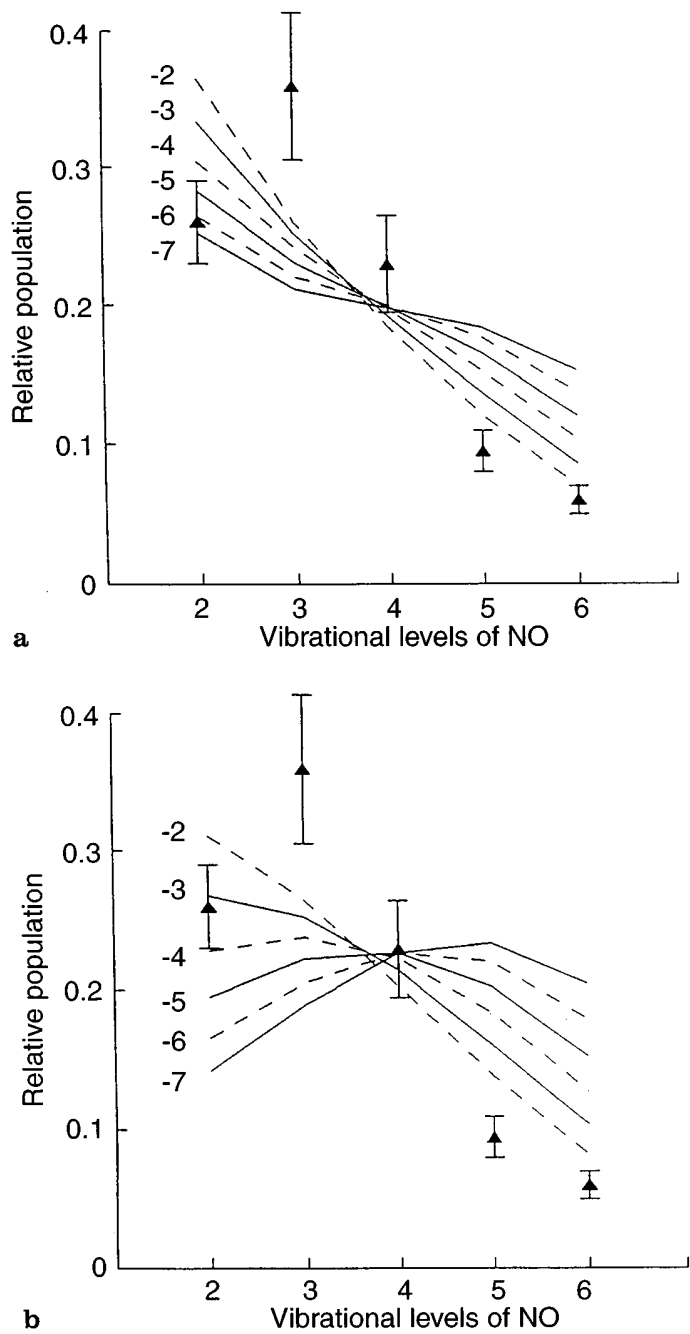

Fig. 3a,b. The comparison of calculated NO populations at an altitude $98 \mathrm{~km}$ for $\lambda_{1}=-7--2$ (solid and dashed lines) with the data of scan 137 of Rawlins et al. (1981) (triangles): a $n=1$, b $n=12$ 
measurement, due to uncertainties in the apparatus calibration, was estimated to be a factor of 2.5.

The enhanced atomic oxygen concentrations are in disagreement with the estimations from the analysis of seven scans for the value $\lambda_{1}$ according to Rawlins et al., (1989) (Fig. 2a,b). Moreover, the predicted distribution for enhanced $[\mathrm{O}]$ and $\lambda_{1}=-4$ is considerably broader than the measured one. We suggest that the disagreement can be related to a stronger vibrational dependence of the rate of the reaction (5). On the other hand, the absence of vertical attitude stabilization in the rocket experiment of Rawlins et al. (1981) could lead to the additional uncertainties in the measured $[\mathrm{NO}(v)]$ distributions. The payload has pitched at the rate of 10.5 degrees/s and this caused the interferometer's field of view to scan across the sky.

Also the sum of energy efficiencies of chemiluminescent mechanisms shown in Eqs. (1) and $(2 \mathrm{a}, 2 \mathrm{~b})$ of 5.3$\mu \mathrm{m}$ emission have been calculated and are presented in Fig. 4a,b. The experimental estimation $1.1 \cdot 10^{-2}$ of
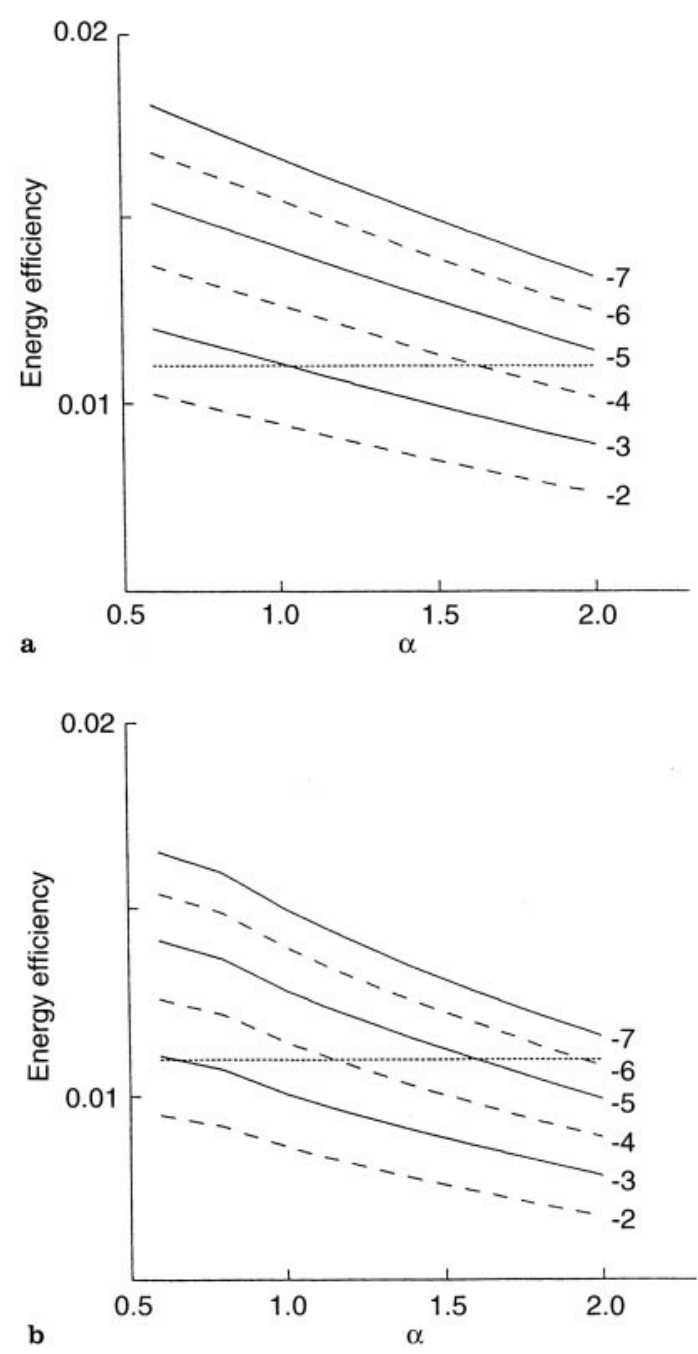

Fig. 4a,b. The comparison of sum of calculated energy efficiencies of mechanisms (1) and (2a, 2b) for $\lambda_{1}=-7--2$ (solid and dashed lines) with experimental estimation of Rawlins et al. (1981) (dotted line): a $n=1, \mathbf{b} n=12$
Rawlins et al. (1981) is shown here. It is seen to exceed sufficiently our calculation for $\lambda_{1}=-6$ and [O] according to MSIS- 83 over the experimental estimation. The calculation agrees better with the estimated efficiency $1.5 \cdot 10^{-2}$ of Reidy et al. (1982). Since the predicted energy efficiencies depend on the production and loss rates of $\mathrm{N}\left({ }^{4} \mathrm{~S}\right)$ and $\mathrm{N}\left({ }^{2} \mathrm{D}\right)$ in the chemical reactions (Torr and Torr, 1982) and by auroral electron impact (Gordiets and Konovalov, 1991), the agreement of our calculation with the estimations of Rawlins et al. (1981) and Reidy et al. (1982) could be considered satisfactory.

We have only varied in the calculations the branching ratios of the reaction $\mathrm{N}\left({ }^{2} \mathrm{D}\right)$ with molecular oxygen. The calculations of energy efficiencies of $5.3-\mu \mathrm{m}$ production mechanisms in Aladjev and Kirillov (1993), and Kirillov and Aladjev (1995) have shown that the contribution of the reaction (1) in the infrared radiation of nitric oxide is sufficiently less than that of the reaction $(2 a, 2 b)$. Torr et al. (1993) and Zipf et al. (1980) have determined that reaction (2b) makes a relatively minor contribution to the thermospheric dayglow and aurora. The results of our calculation show that the contribution of the reaction in $5.3-\mu \mathrm{m}$ emissions is less that that of $\mathrm{N}\left({ }^{2} \mathrm{D}\right)$ reaction. Therefore, we have neglected the change of the branching ratios in the interaction of $\mathrm{N}\left({ }^{4} \mathrm{~S}\right)$ and $\mathrm{N}\left({ }^{2} \mathrm{P}\right)$ with $\mathrm{O}_{2}$ taken according to the experimental estimations of Winkler et al. (1986) and Rawlins et al. (1989).

\section{Conclusions}

The vibrational distribution of nitric oxide in polar ionosphere computed according to one-dimensional non-steady model of chemical and vibrational kinetics of upper atmosphere has been compared with experimental data of seven scans from rocket measurements of Rawlins et al. (1981). Three input parameters of the model have been varied: (a) the surprisal parameter $\lambda_{1}=-7--2$ of $\mathrm{O}\left({ }^{1} \mathrm{D}\right)$ branch in NO production mechanism (Eq. 2a), (b) two regimes of vibrational relaxation in collision of two radicals (Eq. 5) considering the suggestion of a statistical adiabatic model $(n=1)$ or preferable $\Delta v=v_{i}$ transitions $(n=12)$, and (c) the concentrations of atomic oxygen displacing the profile of MSIS-83 and multiplying by a factor of $0.6-2$.

It is determined that the least-averaged deviation of our calculations for both regimes of vibrational deactivation (Eq. 5) from experimental measurements depends on both the surprisal parameter $\lambda_{1}$ and the profile of atomic oxygen concentration. The best agreement for MSIS-83 profile was obtained for $\lambda_{1}=-7--6$ where $n=1$ and $\lambda_{1}=-6--5$ when $n=12$. The values of the surprisal parameter are in better agreement with laboratory estimations of Rawlins et al. (1989). The simultaneous co-ordinated measurements of NO vibrational population and the composition of polar upper atmosphere should determine more exactly the value of $\lambda_{1}$.

The registered depression in some scans of Rawlins et al. (1981) in the population of level $v=2$ and the reduction of the population with the rise of $v \geq 3$ can be obtained in our calculation if enhanced by a factor of 
2-3 atomic oxygen concentration, $n=12$ and $\lambda_{1} \approx-4$ are taken. The conclusion is in disagreement with the result of the comparison of our calculation with those registered in seven scans of NO vibrational population. Perhaps the depression can be explained by a stronger vibrational dependence of the rate of the reaction (5) and the absence of vertical attitude stabilization in the rocket experiment of Rawlins et al. (1981).

Thus, the exact knowledge of the rate coefficients of nitric oxide production and deactivation mechanisms should allow us to estimate the atomic oxygen concentration from the observation of infrared NO emission. Vice versa the co-ordinated measurements of the emissions and atmospheric composition could produce more information on the peculiarities of the chemical and vibrational kinetics of nitric oxide.

Acknowledgements. This work is supported by grant 95-05-15732 from the Russian Foundation of the Fundamental Researches. We would like to thank the referees for their helpful comments.

Topical Editor thanks M. J. Lopez-Gonzalez and another referee for their help in evaluating this paper.

\section{References}

Aladjev, G. A., and A. S. Kirillov, The thermospheric infrared cooling in the aurora, Conf. Proc. of 19th Ann. Europ. Meet., IRF Scientific Report, 209, ISSN 0284-1703, 436-439, 1992.

Aladjev, G., and A. Kirillov, Simulation of the thermospheric infrared emissions in the aurora, in Atmospheric Radiation, Ed. K. H. Stamnes, Proc. SPIE 2049, 256-262, 1993.

Bernstein, R. B., and R. D. Levine, Role of energy in reactive molecular scattering: an information-theoretic approach, $A d v$. At. Molec. Phys., 11, 215-297, 1975.

Billingsley, F. P., Calculated vibration-rotation intensities for $\mathrm{NO}\left(\mathrm{X}^{2}\right)$, J. Molec. Spectr., 61, 53-70, 1976.

Caledonia, G. E., and J. P. Kennealy, NO infrared radiation in the upper atmosphere, Planet. Space Sci., 30, 1043-1056, 1982.

Doroshenko, V. M., N. N. Kudryavtsev, and O. V. Yatsenko, Radiation of vibrationally excited $\mathrm{NO}\left(\mathrm{X}^{2}\right)$ molecules in the visible and near IR spectrum region and kinetics of their radiative decay, J. Appl. Spectr. 57, 460-463, in Russian, 1992.

Fernando, R. P., and I. W. M. Smith, Vibrational relaxation of NO by atomic oxygen, Chem. Phys. Lett., 66, 218-222, 1979.

Glänzer, K., and $\mathbf{J}$. Troe, Vibrational relaxation of NO in collisions with atomic oxygen and chlorine, J. Chem. Phys., 63, 43524357, 1975.

Gordiets, B. F., and V. P. Konovalov, Excitation and ionization of ionospheric gas by high-energy electrons, Geomag. Aeron., 31, 649-656, in Russian, 1991.

Gordiets, B. F., and M. N. Markov, and L. A. Shelepin, The theory of infrared radiation in near-earth space, Trans. FIAN, 105, 771, in Russian, 1978.

Green, B. D., G. E. Caledonia, R. E. Murphy, and F. X. Robert, The vibrational relaxation of $\mathrm{NO}(v=1-7)$ by $\mathrm{O}_{2}, J$. Chem. Phys., 76, 2441-2448, 1982.
Green, B. D., G. E., Caledonia, W. A. M. Blumberg, and F. H. Cook, Absolute production rates and efficiencies of NO in electron-irradiated $\mathrm{N}_{2} / \mathrm{O}_{2}$ mixtures, J. Chem. Phys., 80, 773778, 1984.

Huppi, R. J, and A. T. Stair, Aurorally enhanced infrared emissions, Appl. Opt., 18, 3394-3399, 1979.

Kennealy, J. P., F. P. DelGreco, G. E. Caledonia, and B. D. Green, Nitric oxide chemiexcitation occurring in the reaction between metastable nitrogen atoms and oxygen molecules, J. Chem. Phys., 69, 1574-1584, 1978.

Kirillov, A. S., and G. A. Aladjev, Production and vibrational kinetics of nitric oxide in the disturbed polar thermosphere, Adv. Space Res., 16, (1) 105-(1)108, 1995.

Lie-Svendsen, $\varnothing$., M. H. Rees, K. Stamnes, and E. C. Whipple, Jr., The kinetics of 〈hot〉 nitrogen atoms in upper atmosphere neutral chemistry, Planet. Space Sci., 39, 929-943, 1991.

Murphy, R. E., E. T. P. Lee, and A. M. Hart, Quenching of vibrationally excited nitric oxide by molecular oxygen and nitrogen. J. Chem. Phys., 63, 2919-2925, 1975.

Nesbet, R. K., Surprisal theory. // in Theoretical chemistry. Theory of scattering, 6B, Ed. D. Henderson, New York, Academic Press, 79-126, 1981.

Nikitin, E. E., Theory of elementary atomic and molecular processes in gases. Oxford, Clarendon, 1974.

Quack, M., and J. Troe, Specific rate constants of unimolecular processes. II. Adiabatic channel model, Ber. Bunsenges. Phys. Chem., 78, 240-252, 1974.

Quack, M., and J. Troe, Vibrational relaxation of diatomic molecules in complex forming collisions with reactive atoms, Ber. Bunsenges. Phys. Chem., 81, 160-162, 1977.

Rawlins, W. T., G. E. Caledonia, J. J. Gibson, and A. T. Stair, Jr., Infrared emission from $\mathrm{NO}(\Delta v=1)$ in an aurora: spectral analysis and kinetic interpretation of HIRIS measurements, J. Geophys. Res., 86, 1313-1324, 1981.

Rawlins, W. T., M. E. Fraser, and S. M. Miller, Rovibrational excitation of nitric oxide in the reaction of $\mathrm{O}_{2}$ with metastable atomic nitrogen, J. Phys. Chem., 93, 1097-1107, 1989.

Reidy, W. P., T. C. Degges, A. G. Hurd, A. T. Stair, Jr., and J. C. Ulwick, Auroral nitric oxide concentration and infrared emission, J. Geophys. Res., 87, 3591-3598, 1982.

Sharma, R. D., Y. Sun, and A. Dalgarno, Highly rotationally excited nitric oxide in the terrestrial thermosphere, Geophys. Res. Lett., 20, 2043-2045, 1993.

Sharma, R. D., V. A. Kharchenko, Y. Sun, and A. Dalgarno, Energy distribution of fast nitrogen atoms in the nighttime terrestrial thermosphere, J. Geophys. Res., 101, 275-281, 1996.

Smith, I. W. M., Vibrational energy transfer in collisions involving free radicals, in Nonequilibrium vibrational kinetics, Ed. M. Capitelli, Berlin, Springer, 113-157, 1986.

Torr, D. G., and M. R. Torr, The role of metastable species in the thermosphere, Rev. Geophys. Space Phys., 20, 91-144, 1982.

Torr, M. R., D. G. Torr, and P. G. Richards, N $\left({ }^{2} \mathrm{P}\right)$ in the dayglow: measurement and theory, Geophys. Res. Lett., 20, 531-534, 1993.

Winkler, I. C., R. A. Stachnik, J. I. Steinfeld, and S. M. Miller, Determination of $\mathrm{NO}(v=0-7)$ product distribution from $\mathrm{N}\left({ }^{4} \mathrm{~S}\right)+\mathrm{O}_{2}$ reaction using two-photon ionization, J. Chem. Phys., 85, 890-899, 1986.

Zipf, E. C., P. J. Espy, and C. F. Boyle, The excitation and collisional deactivation of metastable $\mathrm{N}\left({ }^{2} \mathrm{P}\right)$ atoms in auroras, J. Geophys. Res., 85, 687-694, 1980. 